\title{
Síndrome de Good: associação entre timoma, hipogamaglobulinemia, aplasia pura da série vermelha e parvovírus B19 - um estudo de caso
}

\author{
Good Syndrome: association between timoma, hypogamaglobulinemia, pure red series \\ aplasia and parvovirus B19 - a case study
}

\section{Síndrome de Good: asociación entre timoma, hipogamaglobulinemia, aplasia de la serie roja pura y parvovirus B19 - un estudio de caso}

Rudá Ávila Monteiro ${ }^{1}$, David Isaac Serruya ${ }^{1}$, Jociney José Pedroso da Silva Júnior ${ }^{1}$, Yasmin da Silva Nascimento ${ }^{1}$, Irilane de Alcântara Figueira ${ }^{1}$, Irineu Lopes de Alcântara Júnior ${ }^{1}$, Miguel Rebouças de Sousa $^{1 *}$, Ruan David Rebouças de Sousa ${ }^{1}$, lury José Rego Moura ${ }^{1}$, Thiago Xavier Carneiro ${ }^{1}$ e Silvânia Yukiko Lins Takanashi.

\section{RESUMO}

Objetivo: Relatar o caso de um paciente acometido por Síndrome de Good, detalhando o quadro clínico inicial, evolução da doença, tratamento realizado, bem como os exames laboratoriais e de imagens responsáveis pelo seu diagnóstico. Relato do Caso: Paciente do sexo feminino, 52 anos, com timoma diagnosticado por exame de imagem após quadros infecciosos recorrentes e sucessivas transfusões de concentrados de hemácias por repetidas anemias. Tratada cirurgicamente com timectomia, apresentou ao exame histopatológico um timoma medular. Obteve resolução temporária do quadro anêmico por cerca de um ano. Desenvolveu novamente quadros de anemia e infecção, sendo aventada alguma causa de imunodeficiência subjacente, com investigação e diagnóstico laboratorial de hipogamaglobulinemia e infecção oportunista por parvovírus B19. Tratada com sucesso por meio de infusões de imunoglobulina humana. Considerações Finais: Poucos estudos debatem acerca da Síndrome de Good, dificultando a criação de medidas terapêuticas e embasamento teórico acerca do assunto.

Palavras-Chave: Doença rara, Timoma, Hipogamaglobulinemia.

\section{ABSTRACT}

Objective: To report the case of a patient with Good Syndrome, detailing the initial clinical picture, evolution of the disease, treatment performed, as well as laboratory tests and images responsible for its diagnosis. Case Report: A 52-year-old female patient with thymoma diagnosed by imaging after recurrent infectious conditions and successive red blood cell transfusions due to repeated anemia. Surgically treated with thymectomy, the patient had a medullary thymoma at histopathological examination. Obtained temporary resolution of the anemic condition for about one year. She developed anemia and infection again, and some underlying cause of immunodeficiency was raised, with investigation and laboratory diagnosis of hypogammaglobulinemia and opportunistic parvovirus B19 infection. Successfully treated by human immunoglobulin infusions. Final Considerations: few studies debate about Good Syndrome, making it difficult to create therapeutic measures and theoretical basis on the subject.

Keywords: Rare disease, Thymoma, Hypogammaglobulinemia.

\section{RESUMEN}

Objetivo: Informar el caso de un paciente con Síndrome de Good, detallando el cuadro clínico inicial, la evolución de la enfermedad, el tratamiento realizado, así como las pruebas de laboratorio e imágenes responsables de su diagnóstico. Informe del caso: Una paciente de 52 años con timoma diagnosticada por imágenes después de afecciones infecciosas recurrentes y sucesivas transfusiones de glóbulos rojos debido

1Universidade do Estado do Pará (UEPA), Santarém, Pará, Brasil.

*E-mail: miguelreboucas1@gmail.com

SUBMETIDO EM: 11/2019

ACEITO EM: 12/2019

PUBLICADO EM: $2 / 2019$

REAS/EJCH | Vol.Sup.n.40 | e2289 | DOI: https://doi.org/10.25248/reas.e2289.2020 Página 1 de 7 
a la anemia repetida. Quirúrgicamente tratado con timectomía, el paciente tuvo un timoma medular en el examen histopatológico. Obtuvo resolución temporal de la condición anémica durante aproximadamente un año. Ella desarrolló anemia e infección nuevamente, y se planteó alguna causa subyacente de inmunodeficiencia, con investigación y diagnóstico de laboratorio de hipogammaglobulinemia e infección oportunista por parvovirus B19. Tratado con éxito por infusiones de inmunoglobulina humana. Consideraciones finales: Pocos estudios debaten sobre el Síndrome de Good, lo que dificulta la creación de medidas terapéuticas y bases teóricas sobre el tema.

Palabras clave: Enfermedad rara, Timoma, Hipogammaglobulinemia.

\section{INTRODUÇÃO}

O mediastino é um espaço da cavidade torácica que abriga inúmeras e importantes estruturas que podem ser fontes de neoplasias, para fins de descrição, o mediastino é dividido em partes anterior, média e posterior, contendo na parte anterior, o timo, alguns linfonodos e pode conter extensões da tireoide e paratireoide, na parte média contém o coração, grandes vasos, linfonodos, traqueia e alguns nervos como o frênico e o vago, e na parte posterior contém o esôfago, aorta descendente, ducto torácico, linfonodos e gânglios espinais (DRAKE RL, et al., 2015).

No timo, pode-se desenvolver o timoma, tumor derivado de células epiteliais, considerado o mais frequente dentre todas as neoplasias do mediastino anterior, apesar de ser raro quando comparado a todos os tumores originados no mediastino (FERREIRO A, et al., 2015). O timoma é uma neoplasia pouco frequente, com incidência de 1 a 5 casos por cada 1,5 milhão de pessoas/ano e pode surgir em todas as idades, com um pico de incidência entre 55 e 65 anos, representando cerca de 30\% das neoplasias do mediastino anterior em adultos (RAO AC, et al., 2013)

Esta neoplasia ainda pode cursar com outras condições clínicas, como a miastenia gravis (MG), a hipogamaglobulinemia e Aplasia Pura da Série Vermelha (APSV), no entanto, felizmente somente $5 \%$ dos timomas evoluem com APSV. A APSV é uma síndrome clínica rara caracterizada por séries eritrocitárias normocíticas, normocrômicas, com reticulocitopenia importante $(<10.000 / \mathrm{mL})$ associado com a diminuição ou inexistência de eritroblastos na medula óssea, com presença normal de precursores das outras séries celulares, esta pode se manifestar de maneira aguda e autolimitada, frequentemente observada em crianças e, de maneira crônica, mais comum em adultos (PACHECO LF, 2015).

Em relação as demais condições clínicas associadas, temos primeiramente a miastenia gravis (MG), que é uma doença que afeta a porção pós-sinaptica da junção neuromuscular, afetando, portanto, a transmissão dos impulsos nervosos aos músculos efetores (BRASIL, 2015). Já a hipogamaglobulinemia é uma patologia associada a baixos níveis séricos de anticorpos, estando associadas a um maior número de infeções no indivíduo afetado (BRUGNOLLI RM, et al., 2019).

Cerca de 50\% dos casos de APSV não têm etiologia determinada, com um terço dos casos associado a doenças linfoproliferativas, principalmente leucemia linfocítica e apenas $9 \%$ associado ao timoma e caracterizada por deficiente geralmente total dos glóbulos vermelhos no sangue, causando anemia grave (KLEIN WDP, et al., 2015) A hipogamaglobulinemia se caracteriza por níveis reduzidos de imunoglobulinas séricas e prejuízo na síntese de anticorpos, predispondo às infecções bacterianas de repetição, mesmo após a instituição de terapêutica adequada. Tais infecções são responsáveis, ainda, pelo comprometimento da qualidade de vida dos pacientes, da necessidade frequente de antibioticoterapia e de hospitalizações, além de determinarem uma das principais causas de óbito nesse grupo (PULICI GA, 2006).

Robert Good foi o primeiro pesquisador a reconhecer a associação de imunodeficiências e o timoma no ano de 1954, sendo essa associação denominada Síndrome de Good. Epidemiologicamente, costuma acometer preferencialmente adultos entre 40 a 70 anos de idade, ocorre em 7 a 13\% dos pacientes com hipogamaglobulinemia e a incidência de hipogamaglobulinemia em pacientes portadores de timoma varia entre 6 a 11\%. (SERPA FS, et al., 2015). A raridade da doença, associada à escassez de informações, faz com que ainda existem várias controvérsias em relação ao melhor estadiamento, classificação patológica, 
tratamento, fatores prognósticos e como estes se relacionam com as taxas de sobrevida (BALDOTTO CS, et al., 2008).

\section{RELATO DO CASO}

Paciente EMAM, 52 anos, sexo feminino, parda, professora, brasileira, residindo em Belém-PA e natural de Santarém-PA, iniciou fraqueza ao realizar atividades do cotidiano com cefaleias intensas, palidez cutânea, hipoestesias nos dedos das mãos e síncope, sintomas que a motivaram a procurar atendimento médico em agosto de 2014. No pronto atendimento, foi constatado anemia grave com necessidade de transfusão de concentrados de hemácias e internação hospitalar, sendo estabilizada e encaminhada para consulta especializada em hematologia a nível ambulatorial. Durante um período, a paciente experimentou uma melhora sensível, que, depois, foi modificada e a fez retornar ao ambulatório

$\mathrm{Na}$ investigação diagnóstica, o mielograma observou-se série eritrocítica hipocelularidade absoluta (5\%), já a série megacariocítica hipocelularidade com plaquetopoese conservada e a série granulocítica normocelularidade absoluta (86\%), o que fez o patologista interrogar "anemia aplásica?" (Tabela 1).

Tabela 1 - Resultados divergentes do mielograma (18/08/2015) e da biópsia de medula óssea (26/08/2015) realizados em Belém.

\begin{tabular}{lcc}
\hline & Mielograma & Biópsia \\
\hline Celularidade & Hipocelularidade discreta & $50 \%$ de tecido mielolinfoide e $50 \%$ de tecido adiposo \\
\hline Eritrocítica & $\begin{array}{c}5 \% \text { (hipocelularidade } \\
\text { absoluta) }\end{array}$ & Normocelularidade e normomaturação \\
\hline Granulocítica & $\begin{array}{c}86 \% \text { (normocelularidade } \\
\text { absoluta) }\end{array}$ & Normocelularidade e normomaturação \\
\hline Megacariocítica & $\begin{array}{c}\text { Hipocelularidade com } \\
\text { plaquetopoese conservada }\end{array}$ & Normocelularidade com elementos maduros \\
\hline Linfoplasmocitoide & Normal & $\begin{array}{c}\text { Leve a moderada quantidade intersticial com } \\
\text { formação de agregados intertrabeculares }\end{array}$ \\
\hline
\end{tabular}

Fonte: Monteiro RA, et al., 2019

O estudo imunoistoquímico da biópsia de medula óssea concluiu que "o perfil revelava maturidade medular preservada e moderada linfocitose reacional. Ausência de processo linfoproliferativo. Achados imunoistoquímicos incaracterísticos. Observou-se que os achados não coincidiam com os resultados do mielograma. Após várias tentativas de identificação diagnóstica, sem resultados satisfatórios que indicassem um diagnóstico preciso, o hematologista sugeriu que os exames fossem repetidos em um laboratório particular em São Paulo. A partir desse momento, novembro de 2015, foi fechado o primeiro diagnóstico: Aplasia Pura da Série Vermelha (APSV). O diagnóstico foi concluído a partir de novo mielograma e de outra biópsia que, dessa vez, convergiram para a conclusão diagnóstica, conforme dados da Tabela 2.

Tabela 2 - Resultados concordantes do mielograma (13/11/2015) e da biópsia de medula óssea (13/11/2015) realizados em São Paulo.

\begin{tabular}{lcc}
\hline & Mielograma & Biópsia \\
\hline Celularidade & Normocelular & Normocelular \\
\hline Eritrocítica & Hipocelularidade acentuada & Hipocelularidade acentuada \\
\hline Granulocítica & $\begin{array}{c}\text { Normocelular com escalonamento } \\
\text { maturativo mantido }\end{array}$ & Hipercelular, normomaturativa \\
\hline Megacariocítica & Normocelular. normomaturativa & Normocelular \\
\hline Linfoplasmocitoide & Nada digno de nota & $\begin{array}{c}\text { Alguns maduros, em disposição intersticial } \\
\text { em agregados nodulares intertrabeculares }\end{array}$ \\
\hline Reticulócitos & $\ldots$ & $5.000 / \mathrm{mm}^{3}$ \\
\hline
\end{tabular}

Legenda: $\mathrm{mm}^{3}$ - milímetros cúbicos

Fonte: Monteiro RA, et al., 2019

REAS/EJCH | Vol.Sup.n.40 | e2289 | DOI: https://doi.org/10.25248/reas.e2289.2020 Página $\mathbf{3}$ de $\mathbf{7}$ 
A paciente começou a fazer uso de corticoide - prednisona - $20 \mathrm{mg} / \mathrm{dia}$. Após aproximadamente 3 meses de corticoterapia, ela não obteve evolução favorável, com manutenção da sintomatologia inicial, efeitos colaterais acentuados do remédio, e presença de infecções frequentes, tais como inflamação das gengivas, aftas na boca e faringite, sendo então suspensa a medicação.

Pediu-se então uma Tomagrafia Computadorizada (TC). Após resultado da TC de tórax, foi encaminhada para avaliação em um serviço de Cirurgia Torácica, que indicou intervenção cirúrgica pela alta probabilidade de ser um timoma. A cirurgia foi realizada em fevereiro de 2016 por videotoracoscopia e as condições póscirúrgicas da paciente foram estáveis, permanecendo internada em ambiente semi-intensivo por 3 dias, onde foi melhorando progressivamente.

Por um período de 1 ano e 4 meses, após a cirurgia, EMAM apresentou melhora clínica dos sintomas hematológicos, permanecendo apenas as manifestações de aftas na boca, o que era minimizado com medicações tópicas. Permaneceu esse período em acompanhamento sistemático com a oncologia, fazendo revisões periódicas. No entanto, em junho de 2017, a paciente voltou a sentir astenia, palidez cutânea, associada a bronquites e lesões orais de repetição, novamente necessitando de várias transfusões sanguíneas em decorrência da baixa acentuada da hemoglobina (Tabela 3).

Tabela 3 - Hemograma evidenciando anemia.

\begin{tabular}{|c|c|}
\hline Série vermelha & $19 / 10 / 17$ \\
\hline Hemácias $\left(10 / \mathrm{mm}^{3}\right)$ & 2,17 \\
\hline Hematócrito (\%) & 19 \\
\hline Hemoglobina $(\mathrm{g} / \mathrm{dL})$ & 6,6 \\
\hline RDW (\%) & 16,1 \\
\hline VCM (fL) & 97,8 \\
\hline HCM (pg) & 30,4 \\
\hline $\mathrm{CHCM}(\%)$ & 34,7 \\
\hline \multicolumn{2}{|l|}{ Série branca } \\
\hline Leucócitos $\left(/ \mathrm{mm}^{3}\right)$ & 5.410 \\
\hline Eosinófilos (\%) & 0 \\
\hline Basófilos (\%) & 0 \\
\hline Mielóticos (\%) & 0 \\
\hline Metamielócitos (\%) & 0 \\
\hline Bastões (\%) & 0 \\
\hline Segmentados & 20 \\
\hline Linfócitos (\%) & 68 \\
\hline Monócitos (\%) & 12 \\
\hline Plaquetas $\left(/ \mathrm{mm}^{3}\right)$ & 171.000 \\
\hline
\end{tabular}

\section{Legenda:}

$\mathrm{mm}^{3}$ - milímetros cúbicos;

$\%$ - porcentagem;

$\mathrm{g} / \mathrm{dL}$ - gramas a cada decilitro;

$\mathrm{fL}$ - fentolitros;

pg - picogramas.

Fonte: Monteiro RA, et al., 2019.

Neste período foi reencaminhada para a hematologia e novas investigações laboratoriais foram realizadas, cujos indícios indicavam para mielodisplasia. O próximo passo do hematologista foi encontrar a causa dessa condição. Após várias investigações, como resultado do exame Proteína C-reativa (PCR) uma positividade para parvovírus B19. No exame de eletroforese de proteínas séricas, a presença de hipogamaglobulinemia, 
com uma deficiência acentuada de imunoglobulinas. Esses achados levaram ao seu terceiro e quarto diagnósticos concluídos: infecção por parvovírus B19 e hipogamaglobulinemia, respectivamente (Tabela 4).

Tabela 4 - Eletroforese de proteínas, imunoglobulinas e PCR para parvovírus B19, realizados em dezembro de 2017.

\begin{tabular}{lcc}
\hline Exame & Resultado & Referência \\
\hline Eletroforese de proteínas & Hipogamaglobulinemia & Normal \\
Diga $(\mathrm{mg} / \mathrm{dL})$ & 4,1 & $40-350$ \\
$\operatorname{lgM}(\mathrm{mg} / \mathrm{dL})$ & 1,8 & $50-300$ \\
$\operatorname{lgG}(\mathrm{mg} / \mathrm{dL})$ & 56 & $650-1600$ \\
Parvovírus B19 & Detectado & Não detectado \\
\hline
\end{tabular}

Legenda: $\mathrm{mg} / \mathrm{dL}$ - miligramas a cada decilitro

Fonte: Monteiro RA, et al., 2019

No início do ano de 2018, E.M.A.M., após o diagnóstico de Síndrome de GOOD (SG), começou a receber transfusões de Imunoglobulina Humana, na dosagem de $50 \mathrm{mg}$ no primeiro dia e $50 \mathrm{mg}$ no segundo dia, a cada 30 dias. A administração resultou na eliminação do parvovírus, normalização dos níveis de hemoglobina, ausência de bronquites, e infecções orais, eliminação dos sintomas da astenia, palidez cutânea, consequência da normalização da hemoglobina.

Após essa dose inicial, procedeu-se a diminuição da dosagem para $25 \mathrm{mg}$, endovenosa (EV), dose única, a cada 60 dias. Com essa dosagem, a paciente voltou a ter ocorrências de infecções. Com isso, a dose de Imunoglobulina Humana foi aumentada para $40 \mathrm{mg}$, EV, dose única, a cada 45 dias. Neste caso, em específico, foi necessário a reavaliação das dosagens de acordo com as incidências de infecções.

Atualmente a paciente está em fase de adequação da dosagem de Imunoglobulina, com uma vida normal, trabalhando e mantendo a sua qualidade de vida.

\section{DISCUSSÃO}

A síndrome de Good (SG) é uma síndrome rara, com poucos estudos na literatura mundial, com menos de 200 casos descritos até 2015, ou seja, ainda não existem protocolos de tratamento sistematizados, determinando assim prognósticos variados (MALPHETTES M, et al., 2015). Observando-se as últimas pesquisas realizadas e o caso descrito, destaca-se como importante as considerações em relação ao sexo dos pacientes, idade, diagnóstico, ordem cronológica de surgimento do timoma em relação a hipogamaglobulinemia, principais infecções acometidas, principais doenças autoimunes associadas, tipos histológicos do timoma e o tratamento da SG (JORGE C, et al., 2018).

Nesta pesquisa, a paciente é do sexo feminino. Analisando a literatua, tem-se que não há prevalência do sexo, assim como no presente estudo (JANSEN A, et al., 2016). Constatou-se que a média de idade durante os primeiros sintomas da SG foi de 56 anos (MALPHETTES M, et al., 2015). Nesta pesquisa, a paciente tinha 52 anos no momento do diagnóstico, uma idade que se assemelha as encontradas nas outras pesquisas, em torno da quinta década de vida.

Quando analisada a ordem cronológica do surgimento do timoma em relação a hipogamaglobulinemia,) alguns pesquisadores encontraram que o timoma foi diagnosticado concomitante à hipogamaglobulinemia na maioria dos seus pacientes estudados (MALPHETTES M, et al., 2015; JANSEN A, et al., 2016). Enquanto que em outras pesquisas o diagnóstico de timoma precedeu a hipogamaglobulinemia na maior parte dos pacientes (YANG O, 2010). Por fim, em alguns trabalhos o diagnóstico do timoma se deu, na maior parte dos casos, antes do diagnóstico da hipogamaglobulinemia (JOVEN MH, et al., 2013). 
Os timomas geralmente se apresentam em uma das três principais formas: 1) como um achado incidental na imagem torácica em um paciente assintomático; 2) devido a sintomas locais (torácicos) e sua avaliação subsequente como dor torácica, tosse, falta de ar e paralisia do nervo frênico, entre outros; 3) durante a avaliação de uma síndrome paraneoplásica como MG, APSV, hipogamaglobulinemia, entre outros (TABET $R E, 2016)$. No caso da paciente desta pesquisa, o timoma foi encontrado durante avaliação de uma síndrome paraneoplásica - APSV.

A relação etiológica entre timoma e hipogamaglobulinemia na SG ainda não está clara, embora algumas evidências apontem para um defeito básico na medula óssea. A interrupção de células pré-B, o comprometimento da maturação dos precursores eritróides, distúrbios na diferenciação da linhagem de células $B$ devido a fatores humorais derivados da medula óssea e disfunção das células $T$, causando distúrbios na diferenciação da linhagem de células B, têm sido mecanismos propostos. Todos esses mecanismos são factíveis para predispor pacientes com SG a desenvolver infecções recorrentes (JOVEN $\mathrm{MH}$, et al., 2013).

Tanto o atraso no diagnóstico do timoma quanto da hipogamaglobulinemia, submete o paciente a fatores que podem alterar a morbimortalidade do caso. A paciente do caso foi submetida a inúmeras transfusões sanguíneas até o diagnóstico e tratamento do timoma, o que resultou em um aumento exagerado da sua dosagem de ferritina, correndo o risco de causar uma hemocromatose secundária. Assim, como o atraso no diagnóstico e tratamento da hipogamaglobulinemia a fez ficar susceptível a inúmeros casos de infecções. $O$ diagnóstico e tratamento precoce são essenciais nos pacientes com SG.

A APSV pode ter origem congênita, como no caso da anemia de Diamond-Blackfan, onde ocorrem alterações genéticas que atingem a linhagem eritroide, pode ser também de origem adquirida, como no caso das infecções por parvovírus B19 (FISCH P, et al., 2000), pode ser de origem idiopática, ou seja, de causa desconhecida e ainda em pacientes com timona ou durante utilização de imunossupressores que irão desencadear uma inibição na produção dos eritrócitos como, por exemplo, a ciclosporina e os corticosteroides.

O caso desta pesquisa teve atraso no diagnóstico da Aplasia Pura da Série Vermelha (APSV) por conta de discordâncias em exames laboratoriais, mais especificamente entre mielograma e biopsia de medula óssea. Enquanto no mielograma os resultados eritrocíticos concluíam em hipocelularidade, na biópsia de medula óssea a linhagem eritrocítica resultava em normocelularidade e normomaturação. Ambos os exames tinham demais linhagens celulares sem alterações. Em relação à literatura, tem-se que a APSV não é o diagnóstico mais em amostragens maiores (MALPHETTES M, et al., 2015).

Um estudo obteve como tipo histológico mais frequente de timoma o tipo AB (classificação da Organização Mundial da Saúde), e apenas 2 pacientes tinham o tipo A (YANG O, 2016). Na paciente do caso, após ressecção do tumor e estudo histológico, foram encontrados cortes histológicos que mostraram neoplasia constituída por células fusiformes ou ovaladas com núcleos regulares, exibindo cromatina bem distribuída e por vezes pequenos nucléolos, raras figuras de mitoses e a lesão estava envolvida por cápsula fibrosa, compatível com o tipo A na classificação da OMS e tipo I na classificação de MASAOKA (DETTERBECK FC, et al., 2011).

Até o momento, nenhum protocolo de tratamento definitivo foi estabelecido para SG. A timectomia geralmente previne a invasividade dos timomas e o indicador mais importante de prognóstico a longo prazo é a integridade da ressecção (KELLEHER P; MISBAH SA, 2003). A timectomia possui efeitos favoráveis sobre diversas síndromes paratímicas, como MG e APSV. No entanto, é geralmente ineficaz na melhora do quadro imunológico. Em alguns casos, observou-se que a timectomia poderia piorar a hipogamaglobulinemia (JOVEN $\mathrm{MH}$, et al., 2013). Até o ano de 2015, menos de 200 casos tinham sido descritos na literatura mundial, o que influenciou na inexistência de protocolos de diagnóstico e tratamentos sistematizados. Esta pesquisa enfatizou a importância do diagnóstico precoce, com um investimento nos exames laboratoriais e de imagens, e demonstrou uma forma de tratamento considerada bem-sucedida com o uso da timectomia associada a Imunoglobulina Humana. 


\section{REFERÊNCIAS}

1. BALDOTTO CS, et al. Avaliação Retrospectiva do Tratamento e Sobrevida dos Pacientes com Neoplasia Maligna do Timo no Instituto Nacional de Câncer (INCA). Revista Brasileira de Oncologia Clínica. Vol. 5. N.ำ 15 (Set/Dez) 1417, 2008.

2. BRASIL. Protocolo clínico e diretrizes terapêuticas. Miastenia Gravis. 2015

3. BRUGNOLLI RM, et al. Hipogamaglobulinemia secundária reversível causada por terapia anticonvulsivante em adultos: relato de três casos. Brazilian Journal Allergy and Immunology, v. 3, n. 1, p. 86-88, 2019.

4. DETTERBECK FC, et al. The Masaoka-Koga stage classification for thymic malignancies: clarification and definition of terms. Journal of Thoracic Oncology, 2011, 6.7: S1710-S1716.

5. DRAKE RL, et al. Gray's Anatomia para Estudantes. 2. ed. Rio de Janeiro. Elsevier Brasil, 2015. 1192 p.

6. FERREIRO A, et al. Timoma primário intrapericárdico Estádio IV de um caso infrequente e a relevância de um ótimo tratamento cirúrgico. Cirurgia Cardiovascular, v. 22, n. 1, p. 48-52, 2015.

7. FISCH P, et al. Pure red cell aplasia. British journal of haematology, v. 111, n. 4, p. 1010-1022, 2000.

8. JANSEN A, et al. Prognosis of good syndrome: mortality and morbidity of thymoma associated immunodeficiency in perspective. Clinical Immunology, v. 171, p. 12-17, 2016.

9. JORGE C, et al. Cytomegalovirus retinitis in Good Syndrome. Revista Brasileira de Oftalmologia, v. 77, n. 3, 2018.

10. JOVEN MH, et al. Case report and literature review on Good's syndrome, a form of acquired immunodeficiency associated with thymomas. Hawai'i Journal of Medicine \& Public Health, v. 72, n. 2, p. 56, 2013.

11. KELESIDIS T; YANG O. Good's syndrome remains a mystery after 55 years: a systematic review of the scientific evidence. Clinical immunology, v. 135, n. 3, p. 347-363, 2010.

12. KELLEHER P; MISBAH SA. What is Good's syndrome? Immunological abnormalities in patients with thymoma. Journal of clinical pathology, v. 56, n. 1, p. 12-16, 2003.

13. Klein WDP, et al. Aplasia pura da serie vermelha induzida pelo uso de alfaepoetina. Clinical and biomedical research. Porto Alegre. 2015

14. LACY MQ, et al. Pure red cell aplasia: association with large granular lymphocyte leukemia and the prognostic value of cytogenetic abnormalities. Blood, v. 87, n. 7, p. 3000-3006, 1996.

15. MALPHETTES $M$, et al. Good syndrome: an adult-onset immunodeficiency remarkable for its high incidence of invasive infections and autoimmune complications. Clinical Infectious Diseases, v. 61, n. 2, p. e13-e19, 2015.

16. PACHECO LF. Relato de caso: paciente com aplasia pura de série vermelha (cid. d60. 0) curado com o medicamento homeopático phosphorus. 2015. Tese de Doutorado. ALPHA/APH

17. PULICI GA, et al. Função pulmonar de pacientes com hipogamaglobulinemia. Revista Brasileira de Alergia e Imunopatologia, v. 29, n. 3, p. 133-136, 2006.

18. RAO AC, et al. Pure red cell aplasia following thymothymectomy: a case report. The Malaysian journal of medical sciences: MJMS, v. 20, n. 5, p. 83, 2013.

19. SERPA FS, et al. Síndrome de Good - um relato de caso. Revista Salus, v. 1, n. 1, p 11-16, 2015.

20. TABET RE, et al. Thymoma Presenting as Right-Side Heart Failure in a Young Healthy Patient: A Case Report and Literature Review. Journal of Medical Cases, v. 7, n. 8, p. 315-319, 2016. 